\title{
Factors Associated with Development of Prolonged QRS Duration over 20 Years in Healthy Young Adults: The Coronary Artery Risk Development in Young Adults (CARDIA) Study
}

\author{
Leonard IIkhanoff, MDa ${ }^{a}$ Elsayed Z. Soliman, MD, MSc, MS ${ }^{b}$, Hongyan Ning, MD, MS $^{c}$, Kiang \\ Liu, $\mathbf{P h D}^{\mathrm{c}}$, and Donald M. Lloyd-Jones, MD, ScM ${ }^{\mathrm{a}, \mathrm{c}}$ \\ aDepartment of Medicine, Division of Cardiology, Section of Electrophysiology, and the Bluhm \\ Cardiovascular Institute, Northwestern University Feinberg School of Medicine, Chicago, IL \\ bEpidemiological Cardiology Research Center (EPICARE), Department of Epidemiology and \\ Prevention, and Department of Internal Medicine, Cardiology Section, Wake Forest University \\ Health Sciences, Winston-Salem, NC \\ 'Department of Preventive Medicine, Northwestern University, Feinberg School of Medicine, \\ Chicago, IL
}

\begin{abstract}
Background-Data are sparse describing factors associated with development of prolonged QRS duration (QRSd) from young adulthood to middle age.

Methods-We analyzed 12-lead electrocardiograms (ECGs) from the Coronary Artery Risk Development in Young Adults (CARDIA) study over 20 years. We performed logistic regression to examine associations of baseline (Year 0) or average (Year 0 to Year 20) risk factors with incident prolonged QRSd (QRS > $100 \mathrm{msec})$.
\end{abstract}

Results-We included 2,537 participants (57.2\% women, 44.7\% black, mean age 25 years); 292 (11.5\%) developed incident QRSd >100 msec by Year 20. In univariate analyses, baseline covariates associated with incident QRSd prolongation included white race, male sex, ECGLVMI, and baseline QRSd. Similar results were observed after multivariable adjustment.

Conclusion-We found no long-term associations of modifiable risk factors with incident QRSd $>100$ msec. Men, whites, and those with higher ECG-LVMI and QRSd in young adulthood are at increased risk for incident prolonged QRSd by middle age.

\section{Introduction}

The QRS complex on the standard, resting 12-lead electrocardiogram (ECG) is a manifestation of electrical impulse propagation through the conduction system and the ventricular myocardium. A QRS duration (QRSd) $\unlhd 00 \mathrm{msec}$ has been considered "normal" (1). While prolongation of the QRSd (typically > $120 \mathrm{msec}$ ) has been associated with adverse cardiovascular events, including heart failure, sudden cardiac death, and mortality in older adults (2-4), intermediate degrees of QRSd (100-120msec) in younger, healthier populations also have been associated with increased cardiovascular risk (5). Definitions of QRSd conferring risk have varied, and cutpoints of $>100 \mathrm{msec},>110 \mathrm{msec}$, and $>120 \mathrm{msec}$

Corresponding Author: Leonard Ilkhanoff, MD; Assistant Professor, Electrophysiology Section; Division of Cardiology, Department of Medicine; Northwestern Memorial Hospital; Feinberg Pavilion 8-503; 201 East Huron Street; Chicago, IL 60611; Phone:

312-926-2148; Fax: 312-926-2707; 1-ilkhanoff@ northwestern.edu.

Disclosures: LI: None; EZS: None; HN: None; KL: None; DML-J: None 
have all been associated with adverse cardiovascular disease events, including heart failure (6-9). Therefore, since QRSd appears to increase with age $(6,9)$, in healthier and younger populations, an intermediate QRSd may be appropriate to study and may confer cardiovascular risk.

Most studies examining QRSd have been limited by single time point assessments of ECGs or limited longitudinal follow-up. Furthermore, factors associated with the development of a prolonged QRSd from young adulthood to middle age using serial ECGs and long-term traditional risk factor measurements have not been well described. Recognition of factors associated with the development of a prolonged QRSd may provide insight into the possible modifiers of this prognostically important ECG finding in middle age.

In the CARDIA cohort, we hypothesized that participants with exposure to underlying cardiovascular risk factors, such as hypertension and diabetes, would be more likely to develop incident QRSd >100 msec. Therefore, we assessed longitudinal ECGs obtained over a 20 year period in a young, healthy, biracial population to determine prospective associations of established cardiovascular risk factors, subclinical atherosclerosis disease measures, and left ventricular structural measures by echocardiography with incident QRSd prolongation, defined a priori as QRSd $>100 \mathrm{msec}$. Finally, we sought to determine whether there are sex and race differences in the development of incident QRSd >100 msec.

\section{Methods}

\section{Study Population}

Details of the Coronary Artery Risk Development in Young Adults (CARDIA) study enrollment and follow-up have been published elsewhere (10). Briefly, the CARDIA study began in 1985-1986 with the enrollment of 5,115 black and white participants, aged 18 to 30 years and balanced on sex, education status and age distribution within center. Participants were recruited in Birmingham, AL; Chicago, IL; Minneapolis, MN; and Oakland, CA. The institutional review board at each of the study sites approved the study protocols, and written informed consent was obtained from all participants. Participants were examined at baseline (Year 0) and at follow-up examinations in year 2 (Y2), Y5, Y7, Y10, Y15, and Y20. The CARDIA study has had a high retention rate, with $71.8 \%$ completing the in-person examination at Y20. With the addition of telephone contact with participants who did not attend exams, follow-up has been maintained on $91 \%$ of all baseline participants. For this analysis, we included participants who attended the Y20 examination and who had Y20 ECG ascertainment $(\mathrm{N}=2,572)$.

\section{Data Collection}

ECG Analysis-Our analysis included ECGs obtained at CARDIA examinations at Y0 and Y20. The methodology for ECG analysis has been described previously (11). Briefly, the outcome variable (QRSd) was derived from Y20 ECGs that were acquired using identical electrocardiographs (GE MAC 1200 models, GE Milwaukee, WI) in all of the study sites. Resting 12-lead ECGs sampled at a rate of 500 samples per second were recorded in all participants by strictly standardized procedures. Staff was trained to reduce chest electrode placement errors using the Heartsquare device, thereby reducing interindividual variability. The electronic ECGs stored in the electrocardiographs were transmitted regularly over analog phone lines to the CARDIA central ECG Reading Center, EPICARE, located at Wake Forest University, Winston-Salem, NC, for analysis. The study ECGs were automatically processed (after being visually checked for quality) using the 2001 version of the GE Marquette 12-SL program (GE, Milwaukee, WI). Baseline QRSd was derived from the automated measurements and visually confirmed by trained ECG 
coders at EPICARE. QRSd was automatically measured as a global interval from the earliest detection of depolarization in any lead (QRS onset) to the latest detection of depolarization in any lead (QRS offset). This process starts with signal conditioning (filtering), then proceeds to signal averaging (having all QRSs of the same shape aligned in time), and ends by generating a representative QRS complex from which the QRS onset and offset are identified.

A variety of cutpoints for QRSd historically have been used (5-9). Consistent with previous published literature reporting cutpoints for QRSd in the general population (6,9), we defined "normal QRS duration" as a QRSd $₫ 00 \mathrm{msec}$ and a "prolonged QRS duration" as a QRSd $>100$ msec. Measurement of QRSd, therefore, was consistent across examination years. In addition to measurements of QRSd, all CARDIA ECGs were read and coded using the Minnesota Code (MC) ECG classification system (12). Furthermore, sex and race-specific measures of ECG-LV mass (LVM), which have previously been shown to have the closest correlation with echocardiographic LVM (13), were also used and indexed to height ${ }^{2.7}$. Using sex and race-specific calculations incorporating Cornell voltage and body weight devised by Rautaharju (13), values for ECG-LVM index (ECG-LVMI) were calculated. Participants with prolonged QRSd at baseline (Y0 QRSd >100 msec) were excluded from the analysis $(n=35)$.

Clinical Covariates-Clinical covariates from Years 0 and 20, selected a priori because of their known association with cardiovascular diseases, included age, sex, race, measures of blood pressure, height and weight, body-mass index (BMI), smoking history, serum creatinine, education level, and total and HDL-cholesterol. Race and sex were reported by the study participants. Weight was measured with the use of a standard balance-beam scale, with the participant wearing light clothing without shoes. The body-mass index was calculated as the weight in kilograms divided by the square of the height in meters. Diabetes was considered to be present if the person was taking medication for diabetes. Total cholesterol and high-density lipoprotein (HDL) cholesterol were measured from fasting samples as previously described (14). Measures of subclinical atherosclerosis, including coronary artery calcium (CAC) scoring (using computed tomography) and carotid intimal media thickness (IMT) measurements (using B-mode ultrasound) were performed at the Y20 examination (15). These measures were not available at the Y0 (baseline) examination. We, therefore, used CAC and CIMT measures at Y20 examination.

Echocardiographic Imaging-As part of the examination at Y5, CARDIA participants underwent two-dimensional and M-mode echocardiography performed on an Acuson cardiac ultrasound machine (Siemens). Echocardiographic measures of wall thickness and internal dimensions of the left ventricle were performed using standard echocardiographic definitions. Echocardiographic measures in our analysis (at Y5) included left ventricular internal dimensions in diastole and systole, and left ventricular posterior wall thickness in diastole.

\section{Statistical Analysis}

All analyses were performed using SAS version 9.1 (SAS institute, Cary, NC). Characteristics were compared by the QRS status at Y0 and Y20, respectively, among participants using chi-square tests for categorical variables and tests or Wilcoxon rank-sum tests for continuous variables, as appropriate. We compared differences in 20 -year incidence of prolonged QRSd between sex-race groups using chi-square tests. Logistic regression models were fitted to determine the association of incident QRSd prolongation (new QRSd $>100 \mathrm{~ms}$ at Y20) with the covariates. All regression models were adjusted for age, sex, race and clinical covariates (systolic and diastolic blood pressure, serum creatinine, body mass 
index, smoking status, total and high-density lipoprotein cholesterol, hypertension treatment status and ECG left ventricular mass index (ECG-LVMI) in Model 1. To determine whether measures of subclinical atherosclerosis or cardiac structure might mediate the association of established risk factors with prolonged QRSd, in secondary analyses, we further adjusted for CAC presence (defined as CAC score $>0$ ) and CC-IMT at Y20, in addition to echocardiographic variables (left ventricular internal dimension in diastole and posterior wall thickness in diastole) measured at Y5 (Model 2). To determine whether baseline or long-term risk factors were associated with QRSd prolongation, in separate models, we examined two sets of clinical risk factors: "baseline covariates" (from Y0) and "average covariates," which were taken as the average level of measurements across the whole follow-up period, from Y0 to Y20. To be included in the models examining average covariates, participants had to have measurements of each risk factor at Y0 and Y20 and at least one intervening examination. In these analyses, the risk factor levels at Y20 were used for categorical covariates of smoking status, diabetes status and hypertension treatment. Models were also adjusted for baseline (Y0) QRSd. Finally, we determined the change in QRSd from Y0 to Y20 for all participants and for those who had incident QRSd >100 msec. We performed linear regression models using clinical covariates and adjustments as described above, but we used change in QRSd (delta QRSd) as the dependent variable of interest. A two-tailed $\mathrm{p}$ value $<0.05$ was considered statistically significant.

\section{Results}

\section{Study Participants}

There were 2,537 participants included in the study sample who had no QRS $>100$ at baseline (Y0) ECG examination, with 292 (11.5\%) developing incident QRS >100 msec by Y20. Baseline characteristics are shown in Table 1, stratified by QRSd at Y20. White race, male sex, elevated serum creatinine, elevated systolic blood pressure, lower HDL cholesterol, higher BMI, and higher ECG LVMI at Y0 were present in participants with incident QRSd > $100 \mathrm{msec}$ by Y20. In addition, Year 5 echocardiogram parameters of greater LV internal dimension in diastole and a thicker LV posterior wall dimension in diastole were more likely to be present in participants with incident prolonged QRSd by Y20.

Among those with incident QRSd > $100 \mathrm{msec}$, there were 10 with complete and 16 with incomplete RBBB, none with complete and 212 with incomplete LBBB using Minnesota Code criteria. We observed no significant differences in prevalence of other Y20 ECG findings including high amplitude R waves (MC3) and minor ST-T wave abnormalities (MC 4-3, 4-4, 5-3, 5-4) and first degree AV block. However, there was significantly greater prevalence of major STTA (MC 4-1, 4-2, 5-1, 5-2): 6.5\% of those with incident QRSd prolongation had major STTA compared with 3.6\% of those without incident QRSd prolongation $(\mathrm{P}=0.02)$. Among those who did not develop incident $\mathrm{QRSd}>100 \mathrm{msec}$ by Y20, QRSd at Y0 and Y20 were $81.1 \pm 7.0 \mathrm{msec}$, and $87.0 \pm 7.5 \mathrm{msec}$, respectively. Among those who did develop QRSd >100 msec by Y20, QRSd at Y0 and Y20 were 87.2 \pm 7.6 $\mathrm{msec}$, and $107.5 \pm 7.5 \mathrm{msec}$, respectively. The QRSd change between Y20 and Y0 was 5.91 $\mathrm{msec} \pm 8.17 \mathrm{msec}$ for those without incident QRSd > $100 \mathrm{msec}$ at Y20. The QRSd change between Y20 and Y0 was $20.36 \pm 10.37 \mathrm{msec}$ with incident QRSd > $100 \mathrm{msec}$.

\section{Incidence of Prolonged QRSd (>100 msec) over 20 Years}

Figure 1 shows the incidence rates of QRSd $>100 \mathrm{msec}$ stratified by race and sex. Overall, whites were more likely than blacks $(\mathrm{P}<0.001)$ and men were more likely than women $(\mathrm{P}<0.001)$ to develop incident prolonged $\mathrm{QRSd}$. White men were more likely than black 
men to develop incident prolonged $\mathrm{QRSd}(\mathrm{P}=0.004)$. White women also were more likely to develop incident $\mathrm{QRS}>100 \mathrm{msec}$ than black women $(\mathrm{P}=0.023)$.

\section{Associations with Incident QRSd Prolongation at Y20}

Table 2 shows the results of our multivariable analyses examining associations between baseline (Y0) covariates and incident QRSd > $100 \mathrm{msec}$ at Y20. White race, ECG-LVMI at $\mathrm{Y} 0$ and QRSd at Y0 were directly associated with QRS >100 msec at Y20, while female sex was inversely associated (Model 1). In secondary analyses, we examined whether measures of subclinical atherosclerosis, measured at Y20, and echocardiographic parameters of left ventricular structure at Y5, were associated with QRSd >100 msec at Y20. After adjustment for measures of subclinical atherosclerosis at $\mathrm{Y} 20$ and echocardiographic covariates measured at Y5, white race, ECG-LVMI and QRSd at Y0 remained significantly associated with incident QRSd at Y20, and the echocardiographic measure of LV internal dimension in diastole was also significantly associated with incident QRSd at Y20, while LV posterior wall thickness in diastole was of borderline significance, and CAC and CC-IMT were not associated. Sex was no longer associated after adjustment for left ventricular structural variables. When we repeated the analyses examining factors associated with incident QRSd $>110 \mathrm{msec}(\mathrm{N}=53)$, the results were very similar.

In order to determine whether patterns of association might differ for long-term levels of risk factors, rather than single baseline measures, we examined associations between "average" clinical covariates from Y0 to Y20 in separate models (Table 3). Overall patterns and magnitudes of association were similar to the findings for baseline covariates in primary (Model 1) and secondary (Model 2) analyses.

In linear regression models in which the dependent variable of interest was change in QRSd from Y0 to Y20, we found a similar pattern of results after adjusting for baseline traditional risk factors plus QRSd at Year 0, and after adjusting for average risk factors from Y0 to Y20 (data not shown). In these models, white race, male sex, smoking status, antihypertensive medication use, ECG-LVMI and QRSd at Y0 were associated with absolute change in QRSd over 20 years.

\section{Analyses by Sex-Race Groups}

We observed no significant interactions between covariates associated with incident QRSd prolongation and sex or race. Thus, overall patterns of association were similar for diverse sex-race groups, even though incidence rates differed.

\section{Discussion}

In this analysis of longitudinal risk factor associations with incident prolongation of the QRS complex among young adults from the CARDIA study, we observed two important findings. First, we observed a $11.5 \%$ incidence of QRSd >100 msec over 20 years in a young, healthy, biracial cohort. Moreover, we identify factors that are associated with the development of a prolonged QRSd in this population. Specifically, we found that white race, male sex, and baseline ECG-LVMI and baseline QRSd were significantly associated with incident QRSd prolongation. In addition, echocardiographic measures of LV structure (LV internal dimension and posterior wall thickness at Y5) were generally associated with incident QRSd $>100 \mathrm{msec}$ over 15 to 20 years of follow-up. Findings were similar whether we used baseline risk factor levels from young adulthood or long-term (20-year) "average" risk factor levels. Second, our findings suggest that women and blacks appear to be less susceptible, and men and whites more susceptible, to prolongation of the QRS complex from young adulthood to middle age. Taken together, given the prognostic significance of a 
longer QRSd in middle age, additional studies examining potential sex and race/ethnicity differences linking longer-term cardiovascular risk factor exposures and development of incident QRSd prolongation are warranted.

\section{Prior Studies}

\section{QRS Duration and Cardiovascular Events}

Prior studies from predominantly Caucasian samples have shown that prevalent QRSd predicts cardiovascular events in patients after myocardial infarction, with left ventricular systolic dysfunction, or heart failure (2-8). Fewer studies have examined this ECG phenotype and its prognostic significance in community-dwelling individuals or in diverse racial groups. Reports have been mixed. One prior analysis (16), including Framingham participants with ages ranging from 44 to 78 years of age, reported that age-adjusted incidence of myocardial infarction, angina pectoris, and coronary death were unrelated to baseline QRSd prolongation in both sexes. Conversely, in a large Veteran's Administration study (5) of over 45,000 patients with an average age of $56 \pm 15$ years ( $90 \%$ males), investigators found that every $10 \mathrm{msec}$ prolongation of the QRSd was associated with an $18 \%$ increase in risk for cardiovascular death. Importantly, in patients without bundle branch block or paced rhythms, an increase in QRSd from $\Delta 10 \mathrm{msec}$ to $>130 \mathrm{msec}$ was associated with a $180 \%$ increased risk of cardiovascular death. Recently, investigators (7) reported that persistence of or the development of a prolonged QRSd, defined as a QRSd $\geq 110 \mathrm{msec}$, during antihypertensive treatment, was associated with twice the likelihood of new-onset heart failure, independent of blood pressure lowering, treatment modality, and regression of ECG LVH in patients with essential hypertension. These same investigators have also reported that QRSd predicts mortality and, importantly, sudden cardiac death, in hypertensive patients on intensive medical therapy (8). Therefore, a prolonged QRSd appears to have prognostic implications for development of heart failure, and in predicting overall mortality and sudden death.

\section{Associations of QRS Duration with Cardiac Structural and Functional Measures}

Dhingra et al. evaluated cross-sectional associations with QRSd in middle-aged and older participants of the Framingham study (9). Left ventricular mass, end-diastolic dimension, and left ventricular wall thicknesses were directly associated with prolonged QRSd, whereas fractional shortening (FS) was inversely associated ( $<<0.001)$. There was a significant trend for increasing LV mass and chamber dimensions and decreasing FS across categories of QRS duration, which included QRSd $<100 \mathrm{msec}$, QRSd 100-119 msec, and QRSd $\geq 120$ msec $(p<0.001)$. Our findings are consistent with those of Dhingra et al. First, we found that ECG-LVMI at baseline was associated prospectively with incident QRSd $>100 \mathrm{msec}$ in younger adults. Second, we report that echocardiography parameters of posterior wall thickness and left ventricular chamber size also were associated with incident QRSd > 100 msec.

However, our findings extend the observations of Dhingra et al. with regard to sex differences. First, we noted a sex difference in incident prolonged QRSd, with women consistently demonstrating less susceptibility to incident QRSd >100 msec. Recent studies suggest that female hearts may adapt to myocardial stressors such as hypertension with a differential pattern of myocyte remodeling (17). This pattern may also be partly driven by exposure to sex steroids, such as estrogen (18), which, in women, may play a protective role in adverse cardiac structural remodeling. Second, our study also benefited from inclusion of white and black participants. We found that whites were significantly more likely to develop incident QRSd >100 msec. Despite prior reports suggesting that blacks are more likely to have greater baseline LV mass, and LVH on ECG (19) and thicker posterior walls on 
echocardiography (20) - factors which may theoretically result in prolonged conduction times -- our study found that whites were more likely to have a prolonged QRSd at Y20. Moreover, a prior study using the CARDIA cohort suggested that long-term exposure to risk factors such as hypertension in blacks were more likely to result in cardiac structural changes and subsequent risk of heart failure (21), largely driven by exposure to hypertension. Given the strong associations between long-term risk factor exposure and heart failure risk (22), as well as associations between QRSd and LV remodeling, our findings suggest a possible "disconnect" between long-term risk factor exposure, cardiac structural and electrical changes and race - particularly because whites were more likely to develop a prolonged QRSd over 20 years of follow-up than blacks. Further studies are needed to determine a potential link between QRSd as a predictor of heart failure and possible racial differences in QRSd as a marker of heart failure risk.

\section{Potential Pathophysiologic Mechanisms}

After multivariable adjustment, we did not observe prospective associations with incident QRSd prolongation for any of the traditional cardiovascular risk factors, whether measured at baseline or as long-term average values. Given CARDIA is a young cohort, we cannot comment on whether these associations would remain non-significant with longer follow-up or in older populations. Rather, we observed that "immutable" characteristics (sex and race, and QRSd at baseline) were associated with incident QRSd prolongation over long-term follow-up. In CARDIA, Year 5 echocardiogram LV internal dimension and LV posterior wall thickness also were associated with QRSd >100 msec at Year 20. These structural findings do not appear to be related to risk factor exposures in this study, although blood pressure differences certainly could explain the structural findings noted on echocardiograms at Y5. One may expect that certain myocardial stressors, such as pressure overload and myocyte hypertrophy, may manifest as changes in left ventricular remodeling as measured by echocardiographic metrics, or by ECG criteria for LV mass. While longitudinal echocardiographic information was not available beyond Y5, we speculate that the higher prevalence of "incomplete LBBB" noted in this study suggests conduction delay reflecting structural changes from increased LV size, mass, and/or hypertrophy. In addition, there may be variable susceptibility to QRSd prolongation, either related to or independent of structural or morphologic changes associated with cardiac remodeling. Studies have suggested potential sex differences in the electrophysiologic susceptibility to prolonged QRSd in a hypertensive treated population (23). There may be additional factors, such as genetics (24), that account for additional differences in these findings. In the LIFE Trial of hypertensive patients with ECG-LVH, echocardiographic findings at baseline and year two suggest that the higher incidence of heart failure in black patients may be mediated via a differential response of LV structure and function to hypertension over time (25). The explanations for racial differences noted in incident $Q R S>100 \mathrm{msec}$ in our study are less clear and require further exploratory research.

\section{Study Implications}

Prolongation of the QRSd is associated with adverse cardiovascular outcomes in middle age. Identification of factors that may influence or modify QRSd over time is, therefore, potentially important and may provide insight into mechanisms for which QRSd prolongation serves as a marker of risk. Further studies over time are needed to confirm whether prolongation of the QRSd is linked to adverse cardiovascular events in this cohort, and whether the identified characteristics are consistently associated with prolongation of the QRSd. Understanding the links between cardiac structural changes over time and cardiac conduction properties manifested by the ECG, such as the QRSd or ECG-LVMI, will be critical in understanding the utility of the ECG in predicting adverse cardiovascular events. 


\section{Limitations}

Our data represent a large cohort but may be subject to some selection bias. Participants who did not attend the Y20 examination, and those who attended but did not undergo ECG ascertainment, were more likely to be black, male and smokers at baseline (11). Other baseline covariates did not differ. We are limited in providing cardiac structural information at Y20, as echocardiograms were only available at Y5. Our QRSd cutoff of $>100 \mathrm{msec}$ may not represent the optimal cutpoint to indicate a substantially prolonged QRSd; however, in younger adults, the likelihood of prolonged QRSd using a higher threshold is low, precluding informative analyses. In our study, only $2 \%$ of participants had QRSd $>110$ to $120 \mathrm{msec}$ and $0.6 \%$ had a QRSd $\geq 120 \mathrm{msec}$ at Y20. Furthermore, our secondary analyses examining QRSd at Y20 as a continuous variable revealed similar associations. Lastly, the high prevalence of incomplete LBBB likely reflects the non-specific criteria used by the Minnesota code for this diagnosis.

\section{Conclusion}

Since QRSd is an important prognostic marker in middle age, understanding factors that modify its progression and potentially preventing its development from young adulthood to middle age may have important implications. Despite long-term exposure to cardiovascular risk factors, we found no modifiable risk factor associations with incident QRSd $>100 \mathrm{msec}$. Men, whites, and those with higher ECG-LVMI and QRSd in young adulthood appear to be at higher risk for development of prolonged QRSd by middle age. Further studies aimed at understanding the potential sex and race differences in the development of a prolonged QRS duration are warranted.

\section{Acknowledgments}

None

Funding Sources: Work on this manuscript was supported (or partially supported) by R01 HL086792 and by contracts: University of Alabama at Birmingham, Coordinating Center, N01-HC-95095; University of Alabama at Birmingham, Field Center, N01-HC-48047; University of Minnesota, Field Center and Diet Reading Center (Year 20 Exam), N01-HC-48048; Northwestern University, Field Center, N01-HC-48049; and Kaiser Foundation Research Institute, N01-HC-48050; from the National Heart, Lung and Blood Institute.

\section{References}

1. Josephson, ME. Clinical Cardiac Electrophysiology: Techniques and Interpretations. 3. Lippincott Williams \& Wilkins; 2002. Chapter 5: Intraventricular Conduction Disturbances; p. 110-139.

2. Iuliano S, Fisher SG, Karasik PE, et al. QRS duration and mortality in patients with congestive heart failure. Am Heart J. 2002; 143:1085-1091. [PubMed: 12075267]

3. Shamim W, Yousufuddin M, Cicoria M, et al. Incremental changes in QRS duration in serial electrocardiograms over time identify high risk elderly patients with heart failure. Heart. 2000; 88:47-51. [PubMed: 12067942]

4. Desai AD, Yaw TS, Yamazaki T, et al. Prognostic significance of quantitative QRS duration. Am J Med. 2006; 119:600-606. [PubMed: 16828632]

5. Aro AL, Anttonen O, Tikkanen JT, et al. Intraventricular Conduction Delay in a Standard 12-Lead Electrocardiogram as a Predictor of Mortality in General Population. Circ Arrhythm Electrophysiol. 2011 Aug 13. Epub ahead of print. 10.1161/CIRCEP.111.963561

6. Dhingra R, Pencina MJ, Want TJ, et al. Electrocardiographic QRS duration and the risk of congestive heart failure. Hypertension. 2006; 47:861-867. [PubMed: 16585411]

7. Okin PM, Devereux RB, Kjeldsen SE, et al. Incidence of heart failure in relation to QRS duration during antihypertensive therapy: the LIFE study. J Hypertens. 2009; 27:2271-2277. [PubMed: 19834342] 
8. Morin DP, Oikarinen L, Viitasalo M, et al. QRS duration predicts sudden cardiac death in hypertensive patients undergoing intensive medical therapy: the LIFE study. European Heart Journal. 2009; 30:2908-2914. [PubMed: 19687165]

9. Dhingra R, Nam BH, Benjamin EJ, et al. Cross-sectional relations of electrocardiographic QRS duration to left ventricular dimensions: The Framingham Heart Study. J Am Coll Cardiol. 2005; 45:685-689. [PubMed: 15734611]

10. Friedman GD, Cutter GR, Donahue RP, et al. CARDIA: study design, recruitment, and some characteristics of the examined subjects. J Clin Epidemiol. 1988; 41:1105-1116. [PubMed: 3204420]

11. Walsh JA 3rd, Prineas R, Daviglus ML, et al. Prevalence of electrocardiographic abnormalities in a middle-aged, biracial population: Coronary Artery Risk Development in Young Adults study. J Electrocardiol. 2010; 43:385e1-e9. [PubMed: 20374967]

12. Prineas, RJ.; Crow, RS.; Blackburn, H. The Minnesota Code manual of electrocardiographic findings. Littleton, MA: John Wright-PSG; 1982.

13. Rautaharju PM, Park LP, Gottdiener JS, et al. Race- and sex-specific ECG models for left ventricular mass in older populations. Factors influencing overestimation of left ventricular hypertrophy prevalence by ECG criteria in African-Americans. J Electrocardiol. 2000; 33:205218. [PubMed: 10954373]

14. Bild DE, Jacobs DR, Liu K, et al. Seven-year trends in plasma low-density-lipoprotein-cholesterol in young adults: the CARDIA Study. Ann Epidemiol. 1996; 6:380-387.

15. Lee D-H, Steffers MW, Gross M, et al. Differential associations of weight dynamics with coronary artery calcium versus common carotid artery intima-media thickness: The CARDIA study. Am J Epidemiol. 2010; 15:180-189. [PubMed: 20519263]

16. Kreger BE, Anderson KM, Levy D. QRS Interval Fails to Predict Coronary Disease Incidence: The Framingham Study. Arch Int Med. 1991; 151:1365-1368. [PubMed: 2064487]

17. Piro M, Della Bona R, Abbate A, et al. Sex-related differences in myocardial remodeling. J Am Coll Cardiol. 2010; 55:1057-1065. [PubMed: 20223363]

18. Mahmoodzadeh S, Eder S, Nordmeyer J, et al. Estrogen receptor alpha up-regulation and redistribution in human heart failure. FASEB J. 2006; 20:926-934. [PubMed: 16675850]

19. Vitelli LL, Crow RS, Shahar E, et al. Electrocardiographic findings in a healthy biracial population. Am J Cardiol. 1998; 81:453-459. [PubMed: 9485136]

20. Chaturvedi N, Athanassopoulos G, McKeigue PM, et al. Echocardiographic measures of left ventricular structure and their relation with rest and ambulatory blood pressure in blacks and whites in the United Kingdom. J Am Coll Cardiol. 1994; 24:1499-1505. [PubMed: 7930282]

21. Bibbins-Domingo K, Pletcher MJ, Lin F, et al. Racial differences in incident heart failure among young adults. N Engl J Med. 2009; 360:1179-1190. [PubMed: 19297571]

22. Cheng S, Xanthakis V, Sullivan LM, et al. Correlates of echocardiographic indices of cardiac remodeling over the adult life course: longitudinal observations from the Framingham Heart Study. Circulation. 2010; 122:570-578. [PubMed: 20660804]

23. Okin PM, Roman MJ, Devereux RB, et al. Gender differences and the electrocardiogram in left ventricular hypertrophy. Hypertension. 1995; 25:242-249. [PubMed: 7843774]

24. Sotoodehnia N, Isaacs A, de Bakker PI, et al. Common variants in 22 loci are associated with QRS duration and cardiac ventricular conduction. Nature Genetics. 2010; 42:1068-1076. [PubMed: 21076409]

25. Okin PM, Kjeldsen SE, Dahlof B, et al. Racial differences in incident heart failure during antihypertensive therapy. Circulation Cardiovasc Qual Outcomes. 2011; 4:157-164. 


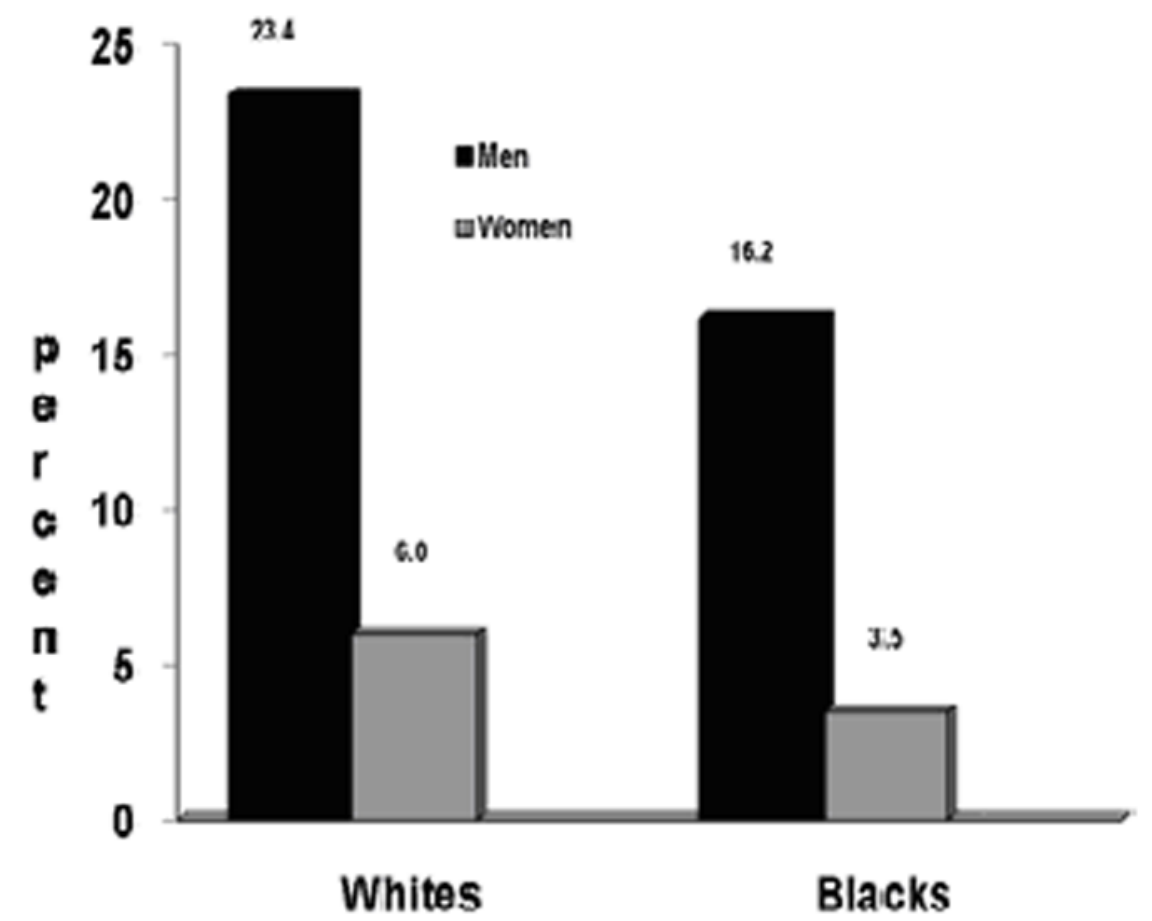

Figure 1. Incidence Rates of QRSd > 100 msec at Year 20 Stratified by Race and Sex Incidence rates are reported as percentage (\%). Comparisons are made between race-sex groups; white men vs. white women $(\mathrm{P}<0.001)$; white men vs. black men $(\mathrm{P}=0.004)$; black men vs. black women $(\mathrm{P}<0.001)$; white women vs. black women $(\mathrm{P}=0.023)$; all blacks vs. all whites $(\mathrm{P}<0.001)$; all men vs. all women $(\mathrm{P}<0.001)$. 


\section{Table 1}

Characteristics of Study Participants Stratified by Incidence of QRSd >100 msec at Y20

\begin{tabular}{|lccc|}
\hline & \multicolumn{3}{c|}{ Incident $\mathbf{Q R S d}>\mathbf{1 0 0}$ msec at Y20 } \\
\hline & No $(\mathbf{n}=\mathbf{2 2 4 5})$ & Yes $(\mathbf{n}=\mathbf{2 9 2})$ & P value \\
\hline Age, year & $\mathbf{2 5 . 1} \pm \mathbf{3 . 6}$ & $\mathbf{2 5 . 3} \pm \mathbf{3 . 4}$ & $\mathbf{0 . 3 5}$ \\
\hline Race, black $(\%)$ & $\mathbf{4 6 . 2}$ & $\mathbf{3 2 . 9}$ & $<\mathbf{0 . 0 1}$ \\
\hline Sex, male $(\%)$ & $\mathbf{3 8 . 5}$ & $\mathbf{7 6 . 0}$ & $<\mathbf{0 . 0 1}$ \\
\hline Y0 Systolic BP $(\mathrm{mmHg})$ & $\mathbf{1 0 9} \pm \mathbf{1 1}$ & $\mathbf{1 1 2} \pm \mathbf{1 0}$ & $<\mathbf{0 . 0 1}$ \\
\hline Y0 Creatinine $(\mathrm{dL})$ & $\mathbf{1 . 0 2} \pm \mathbf{0 . 1 8}$ & $\mathbf{1 . 1 6} \pm \mathbf{0 . 8}$ & $<\mathbf{0 . 0 1}$ \\
\hline Y0 BMI $\left(\mathrm{kg} / \mathrm{m}^{2}\right)$ & $\mathbf{2 4 . 1} \pm \mathbf{4 . 7}$ & $\mathbf{2 5 . 0} \pm \mathbf{5 . 0}$ & $<\mathbf{0 . 0 1}$ \\
\hline Y0 Total Cholesterol $(\mathrm{mg} / \mathrm{dL})$ & $\mathbf{1 7 6 . 7} \pm \mathbf{3 2 . 3}$ & $\mathbf{1 7 8 . 9} \pm \mathbf{3 4 . 1}$ & $\mathbf{0 . 2 8}$ \\
\hline Y0 HDL Cholesterol $(\mathrm{mg} / \mathrm{dL})$ & $\mathbf{5 3 . 9} \pm \mathbf{1 2 . 9}$ & $\mathbf{5 0 . 6} \pm \mathbf{1 2 . 9}$ & $<\mathbf{0 . 0 1}$ \\
\hline Y0 Smoker $(\%)$ & $\mathbf{2 4 . 2}$ & $\mathbf{2 8 . 1}$ & $\mathbf{0 . 1 4}$ \\
\hline Y0 Antihypertensive Therapy $(\%)$ & $\mathbf{2 . 1}$ & $\mathbf{4 . 1}$ & $\mathbf{0 . 0 3}$ \\
\hline Y0 Diabetes $(\%)$ & $\mathbf{0 . 6}$ & $\mathbf{0 . 7}$ & $\mathbf{0 . 9 0}$ \\
\hline Y0 ECG LVMI $\left(\mathrm{g} / \mathrm{m}^{2.7}\right)$ & $\mathbf{3 1 . 9} \pm \mathbf{5 . 2}$ & $\mathbf{3 5 . 1} \pm \mathbf{5 . 8}$ & $<\mathbf{0 . 0 1}$ \\
\hline Y5 Echo LV Internal Dimension in Diastole $(\mathrm{cm})$ & $\mathbf{4 . 9 1} \pm \mathbf{0 . 4 7}$ & $\mathbf{5 . 2 3} \pm \mathbf{0 . 4 7}$ & $<\mathbf{0 . 0 1}$ \\
\hline Y5 Echo LV Posterior Wall Thickness in Diastole $(\mathrm{cm})$ & $\mathbf{0 . 8 3} \pm \mathbf{0 . 1 3}$ & $\mathbf{0 . 8 9} \pm \mathbf{0 . 1 4}$ & $<\mathbf{0 . 0 1}$ \\
\hline Y20 Coronary Artery Calcium Score $>0$ (\%) & $\mathbf{1 6 . 5}$ & $\mathbf{2 6 . 1}$ & $<\mathbf{0 . 0 1}$ \\
\hline Y20 Common Carotid IMT, mm & $\mathbf{0 . 7 9} \pm \mathbf{0 . 1 2}$ & $\mathbf{0 . 8 2} \pm \mathbf{0 . 1 4}$ & $<\mathbf{0 . 0 1}$ \\
\hline & & & \\
\hline
\end{tabular}

Abbreviations: BP: blood pressure; LV: left ventricular; HDL: high density lipoprotein; BMI: body mass index; ECG LVMI: electrocardiographic left ventricular mass index; CAC: coronary artery calcium; IMT: intima medial thickness; Kg: kilograms; m: meters; LVM: left ventricular mass; Echo: echocardiography; Yrs: years; mmHg: millimeters of mercury; dL: deciliter; g: grams; cm: centimeters; \%: percent 
Table 2

Logistic Regression Adjusted for Baseline Traditional Risk Factors Plus QRS at Year 0 and Incident QRS > $100 \mathrm{msec}$ at Year 20

\begin{tabular}{|c|c|c|}
\hline \multicolumn{3}{|c|}{ Odds Ratio (95\% CI) for Incident Prolonged QRS Duration at Year 20} \\
\hline Year 0 Covariates & $\begin{array}{c}\text { Model } 1 \\
(\mathrm{~N}=2501)\end{array}$ & $\begin{array}{c}\text { Model } 2 \\
\text { (N=1911) }\end{array}$ \\
\hline Age (per 1 year) & $0.99(0.95-1.03)$ & $1.01(0.96-1.06)$ \\
\hline White race (vs. Black) & $1.49(1.10-2.02)$ & $1.63(1.11-2.40)$ \\
\hline Female sex (vs. Male) & $0.42(0.28-0.63)$ & $0.71(0.42-1.20)$ \\
\hline $\mathrm{SBP}($ per $1 \mathrm{~mm} \mathrm{Hg}$ ) & $1.00(0.98-1.02)$ & $0.99(0.97-1.01)$ \\
\hline Diabetes & $0.60(0.07-5.01)$ & $0.86(0.09-7.94)$ \\
\hline Creatinine (per $1 \mathrm{mg} / \mathrm{dL}$ ) & $2.14(0.90-5.09)$ & $2.04(0.73-5.67)$ \\
\hline BMI $\left(\right.$ per $\left.1 \mathrm{~kg} / \mathrm{m}^{2}\right)$ & $0.97(0.93-1.01)$ & $0.93(0.88-0.98)$ \\
\hline ECG LVMI (per $1 \mathrm{~g} / \mathrm{m}^{2.7}$ ) & $1.10(1.06-1.14)$ & $1.13(1.08-1.18)$ \\
\hline Current smoker (vs. not) & $1.29(0.95-1.74)$ & $1.20(0.84-1.73)$ \\
\hline Antihypertensive therapy & $2.06(0.95-4.49)$ & $1.90(0.74-4.89)$ \\
\hline QRS duration at Year 0 (per $10 \mathrm{msec}$ ) & $1.09(1.07-1.11)$ & $1.10(1.07-1.12)$ \\
\hline \multicolumn{3}{|l|}{ Additional Covariates } \\
\hline CAC $>0$ at Year 20 & - & $0.94(0.63-1.39)$ \\
\hline Mean maximal CC-IMT at Year 20 & - & $1.52(0.39-5.99)$ \\
\hline Left Ventricular Diastolic Dimension (in mm) & - & $2.11(1.43-3.11)$ \\
\hline Left Ventricular Posterior Wall Thickness (in mm) & - & $3.72(0.99-13.89)$ \\
\hline
\end{tabular}

Abbreviations: SBP: systolic blood pressure; $\mathrm{mm} \mathrm{Hg}$ : millimeters of mercury; mg/dL: milligrams per deciliter; BMI: body mass index; $\mathrm{kg} / \mathrm{m}^{2}$ : kilograms per meter squared; g/m: grams per meter; ECG LVMI: electrocardiographic left ventricular mass index; CAC: coronary artery calcium; CC-IMT: common carotid intima medial thickness 


\section{Table 3}

Logistic Regression Adjusted for "Average" Risk Factors Plus QRS at Year 0 and Incident QRS > 100 msec at Year 20

\begin{tabular}{|c|c|c|}
\hline \multicolumn{3}{|c|}{ Odds Ratio (95\% CI) Incident Prolonged QRS Duration at Year 20} \\
\hline Year 0 Covariates & Model 1 (N=2525) & Model $2(\mathrm{~N}=1936)$ \\
\hline Age (per 1 year) & $0.99(0.96-1.04)$ & $1.00(0.96-1.04)$ \\
\hline White race (vs. Black) & $1.70(1.24-2.31)$ & $1.70(1.24-2.31)$ \\
\hline Female sex (vs. Male) & $0.37(0.25-0.53)$ & $0.37(0.25-0.53)$ \\
\hline SBP per $1 \mathrm{~mm} \mathrm{Hg}$ ) & $1.03(1.00-1.05)$ & $1.03(1.00-1.05)$ \\
\hline Diabetes & $0.60(0.34-1.08)$ & $0.74(0.37-1.49)$ \\
\hline Creatinine (per $1 \mathrm{mg} / \mathrm{dL}$ ) & $1.73(0.97-3.09)$ & $1.73(0.97-3.09)$ \\
\hline BMI $\left(\right.$ per $\left.1 \mathrm{~kg} / \mathrm{m}^{2}\right)$ & $1.02(0.98-1.06)$ & $1.02(0.98-1.06)$ \\
\hline ECG LVMI (per $1 \mathrm{~g} / \mathrm{m}^{2.7}$ ) & $1.03(1.00-1.06)$ & $1.03(1.00-1.06)$ \\
\hline Current smoker (vs. not) & $1.37(0.98-1.92)$ & $1.37(0.98-1.92)$ \\
\hline Antihypertensive therapy & $0.80(0.52-1.22)$ & $0.80(0.52-1.22)$ \\
\hline QRS duration at Year 0 & $1.09(1.07-1.11)$ & $1.09(1.07-1.11)$ \\
\hline \multicolumn{3}{|l|}{ Additional Covariates } \\
\hline $\mathrm{CAC}>0$ at Year 20 & - & $0.96(0.65-1.41)$ \\
\hline Mean maximal CC-IMT at Year 20 & - & $1.34(0.34-5.25)$ \\
\hline Left Ventricular Diastolic Dimension (in mm) & - & $1.88(1.29-2.73)$ \\
\hline Left Ventricular Posterior Wall Thickness (in mm) & - & $4.22(1.16-15.37)$ \\
\hline
\end{tabular}

Abbreviations: SBP: systolic blood pressure; $\mathrm{mm} \mathrm{Hg}$ : millimeters of mercury; $\mathrm{mg} / \mathrm{dL}$ : milligrams per deciliter; BMI: body mass index; $\mathrm{kg} / \mathrm{m}^{2}$ : kilograms per meter squared; g/m: grams per meter; ECG LVMI: electrocardiographic left ventricular mass index; CAC: coronary artery calcium; CC-IMT: common carotid intima medial thickness 